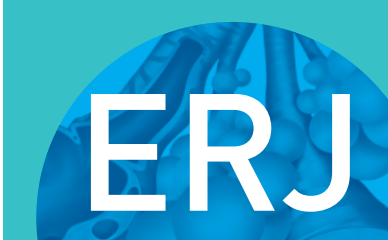

open research

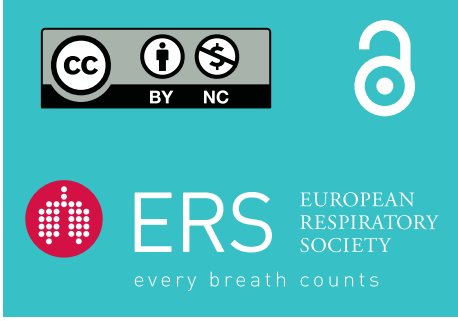

\section{Pulmonary infection caused by Mycobacterium marinum in a patient with anorexia nervosa}

\section{To the Editor:}

The global incidence of nontuberculous mycobacterial pulmonary disease (NTM-PD) has been increasing [1]. Recently popularised microbiology tests, including $16 \mathrm{~S}$ rRNA sequencing and matrix-assisted laser desorption ionisation-time-of-flight mass spectrometry, have made it possible to identify rare nonmycobacterial species previously unidentifiable using conventional methods [2,3]. Transmission of Mycobacterium marinum to humans is mainly through direct contact with domesticated fish or through pre-existing wounds or abrasions on limbs exposed to contaminated water [4]. M. marinum causes swimming pool or fish tank granuloma [4]. The organism grows well at $30-32^{\circ} \mathrm{C}$, but poorly or not at all at $37^{\circ} \mathrm{C}$ [4]. Most M. marinum infections do not invade deeper than the superficial cooler regions of the skin, and pulmonary infections are rarely reported [4].

Anorexia nervosa is characterised by severe restriction of food intake, resulting in severe weight loss and malnutrition [5]. Malnutrition is an important and common risk factor associated with mycobacterial infection by both $M$. tuberculosis and nontuberculous mycobacteria [6]. In addition, increased susceptibility to mycobacterial infections has been reported in individuals with anorexia nervosa $[7,8]$.

A 46-year-old Japanese female office worker was admitted to the psychiatric ward in our hospital because of emaciation. Her physical condition had rapidly deteriorated in the 4 months preceding her admission, although she did not have any specific symptoms other than extreme weight loss. She was a nonsmoker with a 4-year history of restricting-type anorexia nervosa without bulimic episodes and no history of tuberculosis. No abnormalities had been detected in previous annual chest radiographs, suggesting that she had no pre-existing structural lung disease. She reported no recent contact with fish or contaminated water. She had not undergone bacille Calmette-Guérin vaccination. On examination, she was found to be emaciated, weighing $26 \mathrm{~kg}$, with a body mass index (BMI) of $10.2 \mathrm{~kg} \cdot \mathrm{m}^{-2}$ and a body temperature of $35.3^{\circ} \mathrm{C}$. She was not investigated for immunodeficiencies such as HIV infection or endocrine abnormalities. Respiratory symptoms, including cough and sputum, were not evident. Physical examination revealed bronchial breath sounds in both apices. The skin on all four limbs was intact. She did not have peripheral oedema, alopecia or cutaneous pigmentation. A chest radiograph on admission revealed cavities surrounded by infiltrates in both lung apices (figure 1a). Chest computed tomography revealed a large cavitating right upper lobe lesion and multiple thick-walled left upper lobe cavitary lesions (figure 1b). Laboratory tests revealed that the white blood cell count was 4700 cells $\mu \mathrm{L}^{-1}$, with a lymphocyte count of 799 cells $\mu \mathrm{L}^{-1}$, haemoglobin $9.5 \mathrm{~g} \cdot \mathrm{dL}^{-1}$, serum albumin $2.3 \mathrm{~g} \cdot \mathrm{dL}^{-1}$ and serum C-reactive protein $0.6 \mathrm{mg} \cdot \mathrm{dL}^{-1}$.

The patient was initially suspected to have pulmonary tuberculosis and was transferred to the tuberculosis ward. Three out of four forced sputum samples revealed numerous acid-fast bacilli (AFB). She was initiated on $200 \mathrm{mg} \cdot \mathrm{day}^{-1}$ isoniazid, $300 \mathrm{mg} \cdot \mathrm{day}^{-1}$ rifampicin and $500 \mathrm{mg} \cdot \mathrm{day}^{-1}$ ethambutol. A sputum culture grew Mycobacterium that formed smooth photochromogenic colonies that were negative for M. tuberculosis and M. avium complex on PCR assays. M. marinum was identified using a DNA-DNA hybridisation kit (DDH Mycobacteria; Kyokuto, Tokyo, Japan). Analysis of the 16S rRNA gene confirmed

@ERSpublications

Mycobacterium marinum can cause pulmonary infection and can grow at $\leqslant 32^{\circ} \mathrm{C}$. Physicians should consider $M$. marinum when examining patients with pulmonary infection and low body temperature or anorexia nervosa, and grow the specimen at $\leqslant 32^{\circ} \mathrm{C}$. https://bit.ly/3jkzBeq

Cite this article as: Oshima $\mathrm{K}$, Niinuma $\mathrm{Y}$, Saito $\mathrm{H}$, et al. Pulmonary infection caused by Mycobacterium marinum in a patient with anorexia nervosa. ERJ Open Res 2021; 7: 00782-2020 [https://doi.org/10.1183/23120541.00782-2020].

Copyright $\odot$ The authors 2021. This version is distributed under the terms of the Creative Commons Attribution NonCommercial Licence 4.0. For commercial reproduction rights and permissions contact permissions@ersnet.org 

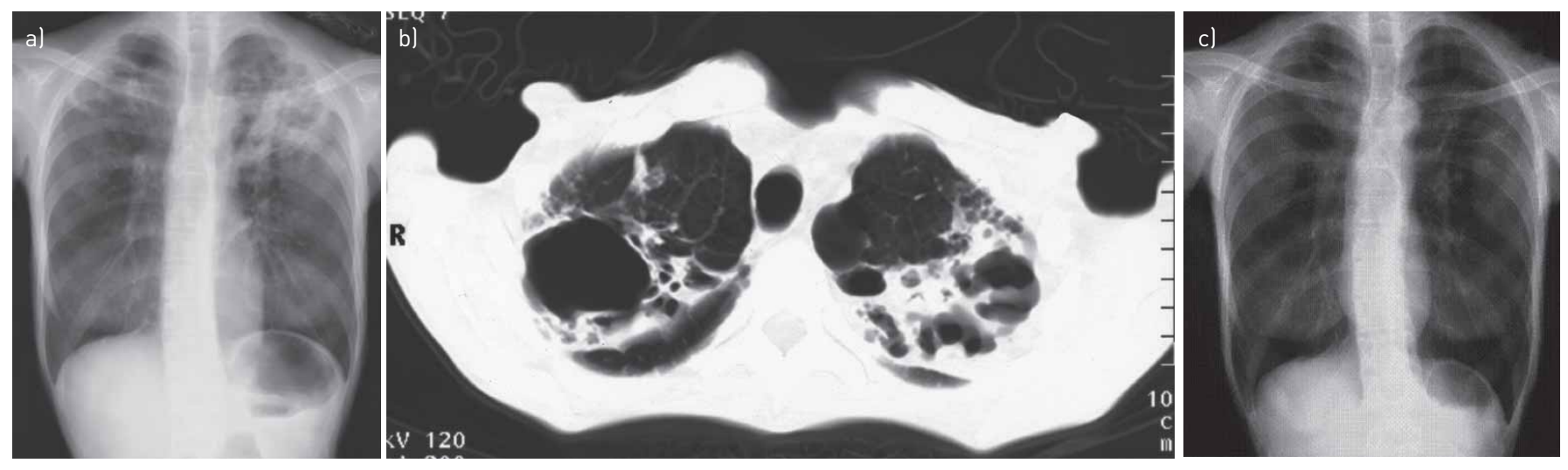

FIGURE 1 Chest imaging showing the patient's pulmonary lesions. a) Chest radiograph before initiating treatment showing bilateral cavities in the upper lobes of the lungs; b) chest computed tomography scan showing bilateral cavitary lesions surrounded by infiltrations in the apices of the lungs; c) chest radiograph 3 months after completing treatment showing a reduction in the size of the apical cavities.

that the mycobacteria was M. marinum, and the isolate was $100 \%$ consistent with that of M. marinum ATCC $927^{\mathrm{T}}$. The primary culture grew on $2 \%$ Ogawa egg medium in a carbon dioxide incubator at $25^{\circ} \mathrm{C}$ and $32^{\circ} \mathrm{C}$, but not at $37^{\circ} \mathrm{C}$ or $42^{\circ} \mathrm{C}$. The minimum inhibitory concentrations of rifampicin and clarithromycin were $\leqslant 1.0 \mu \mathrm{g} \cdot \mathrm{mL}^{-1}$ and $2.0 \mu \mathrm{g} \cdot \mathrm{mL}^{-1}$, respectively. Once the strain had been identified and its drug susceptibility characterised, combination therapy was changed to rifampicin $300 \mathrm{mg}^{-d_{a y}{ }^{-1} \text { and }}$ clarithromycin $200 \mathrm{mg}$ twice daily. The patient's sputum expectoration resolved within several days after she began treatment. After initiating treatment, her body temperature temporarily decreased further and then gradually recovered, suggesting that her body temperature on admission was relatively pyretic due to mycobacterial infection. She was treated with this combination therapy for 6 months along with psychotherapy. A repeat chest radiograph 3 months after completing treatment revealed a reduction in the size of the apical cavities (figure 1c). 9 months after initiating treatment, her weight had increased to $30 \mathrm{~kg}$ and BMI was $11.7 \mathrm{~kg} \cdot \mathrm{m}^{-2}$.

This case reveals four important clinical issues: 1) M. marinum can cause pulmonary infection; 2) it has the potential for acquisition via inhalation; 3) anorexia nervosa may increase susceptibility to pulmonary M. marinum; and 4) $\mathrm{AFB}$ culture at $\leqslant 32^{\circ} \mathrm{C}$ aids in identifying the pathogen.

As of September 2020, only two cases of pulmonary M. marinum infection have been reported in the English-language literature $[9,10]$, and only one of the case reports provided chest images. The radiological finding common to the two cases reported previously and this case is the presence of large cavities with infiltration in lung apices. This may be a characteristic radiological finding associated with M. marinum. In this case, the portal of entry by which the mycobacteria entered the lungs was unidentified, because the patient had no known marine exposure or contact with aquatic animals and did not have any injuries and wounds on the trunk or extremities. This suggests potential airway acquisition rather than direct transmission from an aquatic environment. The growth of $M$. marinum requires several weeks at a low temperature for primary cultivation in vitro [4]. The patient's hypothermia may have developed during the 4 months preceding her admission, providing an environment conducive to growth of the organism. This case suggests that $M$. marinum can grow in the human lung without producing symptoms.

In this case, the patient was extremely emaciated and had multiple risk factors for pulmonary mycobacterial infection. Low BMI is an independent risk factor for NTM-PD [11, 12]. Malnutrition increases susceptibility to pulmonary infection due to altered respiratory anatomy, mainly by reducing the clearance of inhaled pathogens from the airways as a result of respiratory muscle atrophy $[6,11,12]$. Low subcutaneous fat levels decrease production of adipokines, including leptin and adiponectin. Leptin is a hormone produced by white fat cells having immunomodulatory effects on T-lymphocyte differentiation, thereby enhancing phagocytosis and increasing tumour necrosis factor (TNF)- $\alpha$ production. In cases of malnutrition, cytokine is produced predominantly through the type 2 T-helper cell pathway, resulting in increased interleukin 4 production and decreased interferon (IFN)- $\gamma$ production [5]. Because IFN- $\gamma$ and TNF- $\alpha$ play critical roles in the defence against mycobacterial infection [6], individuals with anorexia nervosa would be expected to have an increased susceptibility to mycobacterial infection. It has been reported that patients with certain body morphotypes and immune phenotypes [13], corresponding to those of our patient, are more susceptible to NTM-PD. Furthermore, our patient had hypothermia on 
admission, which may have conferred additional susceptibility because the primary culture of $M$. marinum isolated from the patient's sputum grew at $\leqslant 32^{\circ} \mathrm{C}$.

Anorexia nervosa sometimes presents with hypothermia [14] and the patient's anorexia nervosa-associated hypothermia may have contributed to the growth of $M$. marinum in her lower respiratory tract, leading to pulmonary infection. NTM-PD may produce few symptoms and the radiological changes are variable. Mycobacterium are not routinely cultured at $\leqslant 32^{\circ} \mathrm{C}$. Thus, $M$. marinum infection may be underdiagnosed under standard culture conditions. Physicians should consider M. marinum and culture samples for AFB at $\leqslant 32^{\circ} \mathrm{C}$, especially in patients with hypothermia or an underlying susceptibility to NTM-PD, especially in patients with apical cavitary disease, even if the patient has no known contact with fish or an aquatic environment.

Kengo Oshima ${ }^{1,2}$, Yukari Niinuma ${ }^{3}$, Hajime Saito ${ }^{4}$, Hiroaki Baba ${ }^{1,2}$, Hajime Kanamori ${ }^{1,2,5}$, Tetsuji Aoyagi $^{1,2}$, Koichi Tokuda ${ }^{2,5}$ and Mitsuo Kaku ${ }^{6}$

${ }^{1}$ Dept of Infectious Diseases, Internal Medicine, Tohoku University Graduate School of Medicine, Sendai, Japan. ${ }^{2}$ Dept of Intelligent Network for Infection Control, Tohoku University Graduate School of Medicine, Sendai, Japan. ${ }^{3}$ Ohmichi Internal Medicine and Respiratory Clinic, Sapporo, Japan. ${ }^{4}$ Hiroshima Environment \& Health Association, Health Science Center, Hiroshima, Japan. ${ }^{5}$ Division of Infection Control, Tohoku University Hospital, Sendai, Japan. ${ }^{6}$ Dept of Infectious Disease, Tohoku Medical and Pharmaceutical University, Sendai, Japan.

Correspondence: Kengo Oshima, Dept of Infectious Diseases, Internal Medicine, Tohoku University Graduate School of Medicine, 1-1 Seiryo-machi, Aoba-ku, 980-8574 Sendai, Japan. E-mail: koshima@med. tohoku.ac.jp

Received: 26 Oct 2020 | Accepted after revision: 29 Jan 2021

Acknowledgements: The authors wish to thank the Honyaku Center Company (Osaka, Japan) for editing a draft of this manuscript. No commercial funding was received for this purpose.

Author contributions: K. Oshima wrote the first draft of the manuscript and was responsible for data collection and analysis. Y. Niinuma provided treatment to the patient and was involved in data collection. H. Saito was involved in microbiological analysis. H. Baba, H. Kanamori, T. Aoyagi, K. Tokuda and M. Kaku revised, commented on and corrected this manuscript with their individual expertise. All authors contributed to the writing of the final manuscript and have approved the final draft for publication.

Conflicts of interest: K. Oshima has nothing to disclose. Y. Niinuma has nothing to disclose. H. Saito has nothing to disclose. H. Baba has nothing to disclose. H. Kanamori has nothing to disclose. T. Aoyagi has nothing to disclose. K. Tokuda has nothing to disclose. M. Kaku has nothing to disclose.

\section{References}

1 Brode SK, Daley CL, Marras TK. The epidemiologic relationship between tuberculosis and non-tuberculous mycobacterial disease: a systematic review. Int J Tuberc Lung Dis 2014; 18: 1370-1377.

2 Alcaide F, Amlerová J, Bou G, et al. How to: identify non-tuberculous Mycobacterium species using MALDI-TOF mass spectrometry. Clin Microbiol Infect 2018; 24: 599-603.

$3 \mathrm{Kim} \mathrm{SH}$, Shin JH. Identification of nontuberculous mycobacteria using multilocous sequence analysis of $16 \mathrm{~S}$ rRNA, hsp65, and rpoB. J Clin Lab Anal 2018; 32: e22184.

4 Aubry A, Mougari F, Reibel F, et al. Mycobacterium marinum. Microbiol Spectr 2017; 5: doi:10.1128/ microbiolspec.TNMI7-0038-2016.

5 Gibson D, Mehler PS. Anorexia nervosa and the immune system - a narrative review. J Clin Med 2019; 8: 1915.

6 McShane PJ, Glassroth J. Pulmonary disease due to nontuberculous mycobacteria: current state and new insights. Chest 2015; 148: 1517-1527.

7 Hotta M, Nagashima E, Takagi S, et al. Two young female patients with anorexia nervosa complicated by Mycobacterium tuberculosis infection. Intern Med 2004; 43: 440-444.

8 Hotta M, Minami Y, Itoda I, et al. A young female patient with anorexia nervosa complicated by Mycobacterium szulgai pulmonary infection. Int J Eat Disord 2004; 35: 115-119.

9 Lai CC, Lee LN, Chang YL, et al. Pulmonary infection due to Mycobacterium marinum in an immunocompetent patient. Clin Infect Dis 2005; 40: 206-208.

10 Velu PP, Fernandes SE, Laurenson IF, et al. Pulmonary Mycobacterium marinum infection: "fish tank granuloma" of the lung. Scott Med J 2016; 61: 203-206.

11 Griffith DE, Aksamit T, Brown-Elliott BA, et al. An official ATS/IDSA statement: diagnosis, treatment, and prevention of nontuberculous mycobacterial diseases. Am J Respir Crit Care Med 2007; 175: 367-416.

12 Lake MA, Ambrose LR, Lipman MC, et al. "Why me, why now?" Using clinical immunology and epidemiology to explain who gets nontuberculous mycobacterial infection. BMC Med 2016; 14: 54.

13 Kartalija M, Ovrutsky AR, Bryan CL, et al. Patients with nontuberculous mycobacterial lung disease exhibit unique body and immune phenotypes. Am J Respir Crit Care Med 2013; 187: 197-205.

14 Miller KK, Grinpson SK, Ciampa J, et al. Medical findings in outpatients with anorexia nervosa. Arch Intern Med 2005; 165: 561-566. 